\title{
Pyuria predicts poor prognosis in patients with non-muscle-invasive bladder cancer treated with bacillus Calmette-Guérin
}

\author{
TAKESHI AZUMA, YASUSHI NAGASE and MASAYA OSHI \\ Department of Urology, Tokyo Metropolitan Tama Medical Center, Fuchu, Tokyo 183-0042, Japan
}

Received April 4, 2015; Accepted April 29, 2015

DOI: $10.3892 / \mathrm{mco} .2015 .582$

\begin{abstract}
This study was conducted to evaluate the utility of pyuria as a predictive marker of bacillus Calmette-Guérin (BCG) treatment for non-muscle-invasive bladder cancer (NMIBC). A cohort of 243 NMIBC patients treated with BCG through intravesical instillation between 1994 and 2007 at the Tokyo Metropolitan Tama Medical Center were enrolled in this retrospective study. Pyuria was defined as urine containing $\geq 10$ white blood cells per high-power field. A total of 30 of the patients with pyuria $(30 / 37,81 \%)$ and 87 patients of those without pyuria $(87 / 206,42 \%)$ exhibited recurrence following BCG treatment. The 3-year recurrence-free survival rate of patients with and without pyuria following BCG treatment was 20 and $62 \%$, respectively. The multivariate Cox proportional hazards regression model indicated that pyuria was an independent predictor of disease recurrence following BCG treatment. Patients with inflammatory NMIBC exhibited resistance to therapy with intravesical instillation of BCG.
\end{abstract}

\section{Introduction}

Bacillus Calmette-Guérin (BCG) instillation as an adjuvant to transurethral resection of the bladder tumour (TURBT) is the most effective and widely used therapy for non-muscle-invasive bladder cancer (NMIBC). Although BCG therapy may improve the prognosis of NMIBC patients, $20-40 \%$ patients fail to respond $(1,2)$. BCG treatment commonly induces granulomatous inflammatory changes in the bladder, which produce irritative symptoms. The systemic side effects may vary from mild malaise and fever to life-threatening or fatal sepsis (3). Therefore, it is important to identify a reliable predictive biomarker. Several studies have attempted to identify risk factors for disease recurrence and it was recently demonstrated that certain factors may predict the recurrence of NMIBC (4).

Correspondence to: Dr Takeshi Azuma, Department of Urology, Tokyo Metropolitan Tama Medical Center, 2-9-2 Musashidai, Fuchu, Tokyo 183-0042, Japan

E-mail: tazumauro@gmail.com

Key words: bladder cancer, pyuria, bacillus Calmette-Guérin, predictive factors, recurrence
Although these results are useful for predicting the effectiveness of BCG therapy in various settings, a number of emerging factors have not been considered.

Recently, the association between inflammation and a variety of cancers has been reported $(5,6)$. In our previous study, we demonstrated that pyuria was a prognostic marker of recurrence and progression of newly diagnosed NMIBC (7). The aim of this study was to evaluate the utility of pyuria as a predictive marker of BCG treatment for NMIBC.

\section{Patients and methods}

Study group. Between 1994 and 2007, 243 patients were diagnosed with NMIBC based on histopathological evaluation and were treated with intravesical instillation of BCG following TURBT at the Tokyo Metropolitan Tama Medical Center (Tokyo, Japan).

Urine analysis. Urine analysis for pyuria was performed prior to TURBT and a sample for urine culture was collected at the same time. The cases with NMIBC confirmed by pathological examination underwent BCG instillation. Pyuria was defined as $\geq 10$ white blood cells per high power field of urine (8).

Individual patient data, including number of tumors, tumor size, tumor grade [1973 WHO classification (9)], pathological T stage, presence of concomitant carcinoma in situ and intravesical therapy, were obtained from patient medical records.

Treatment. Instillation with 40,80 or $81 \mathrm{mg}$ BCG (Tokyo or Connaught strain were obtained from Japan BCG or Nippon Kayaku, Tokyo, Japan, respectively) diluted in $40 \mathrm{ml}$ saline was performed between 14 and 28 days after TURBT. The instillation was repeated once weekly for 6-8 consecutive weeks. None of the patients received maintenance instillation. During follow-up, all the patients underwent cystoscopy, urine analysis and cytology every 3 months following BCG treatment.

Statistical analysis. The time-to-recurrence following BCG treatment was defined as the time from the date of the first instillation of BCG to the date of bladder cancer recurrence. Patients who remained alive without recurrence, or those who succumbed prior to recurrence, were censored at the date of the last available follow-up cystoscopy. 
The distribution of recurrence-free survival (RFS) was constructed using the Kaplan-Meier method. The association between survival and each parameter was analyzed with the log-rank test. Univariate and multivariate Cox proportional hazards regression models were summarized with risk ratios and $95 \%$ confidence intervals. The statistical analyses were performed using the JMP ${ }^{\circledR}$ software package. A P-value $<0.05$ was considered statistically significant.

\section{Results}

Patient characteristics. The characteristics of the patients and their tumors are summarized in Table I. Of the 243 patients treated with BCG instillation, 117 (48\%) developed subsequent recurrence. In this analysis, we assessed the presence of pyuria at the time of recurrent tumor resection, prior to BCG treatment. Pyuria was detected in 37 of the 243 patients (15\%) treated with BCG. Recurrence survivors were followed up for a median period of 10 months. The RFS rates at 1 and 3 years were 72 and $56 \%$, respectively. The median time of RFS was 45 months.

Tumor recurrence. Tumor recurrence was diagnosed in $30(81 \%)$ of the 37 patients with pyuria and $87(42 \%)$ of the 206 patients without pyuria following BCG treatment. The RFS rates at 3 years were significantly lower among patients with pyuria vs. those without (20 vs. $62 \%$, respectively; $\mathrm{P}<0.0001$ ) (Fig. 1). The univariate regression analysis revealed that pyuria was associated with RFS following BCG therapy. Specifically, patients with pyuria appeared to be resistant to BCG treatment. The previously reported factors (age, gender, tumor number, tumor size, $\mathrm{T}$ stage and grade) were also associated with RFS following BCG treatment (Table II). Prior recurrence rates were not considered, since only newly diagnosed patients were included in this study. The multivariate regression analysis revealed that pyuria was an independent predictor of tumor recurrence following BCG treatment (Table II). In this model, age, tumor number and size were also identified as independent predictors of tumor recurrence following BCG treatment (Table II).

BCG relapsing was further defined by time of recurrence as early (within 12 months), intermediate (12-24 months) or late (>24 months) (10). Recurrence within 12 months was observed in $21(68 \%)$ of the 30 recurrent cases with pyuria and in only $43(50 \%)$ of the 87 recurrent cases without pyuria. Recurrence after 24 months was observed in 1 (3\%) of the 30 recurrent cases with pyuria and in only 20 (23\%) of the 87 recurrent cases without pyuria (Fig. 2). These data suggested that pyuria was associated with early recurrence following BCG treatment.

\section{Discussion}

Recent studies reported that inflammation within the tumor microenvironment may promote the growth and invasion of cancer cells through an increase in cell proliferation, survival, migration and angiogenesis (11). In our previous study, we demonstrated that NMIBC with pyuria was associated with poor clinical prognosis and that the presence of the pyuria could be used as an indicator of recurrence and progression (7).

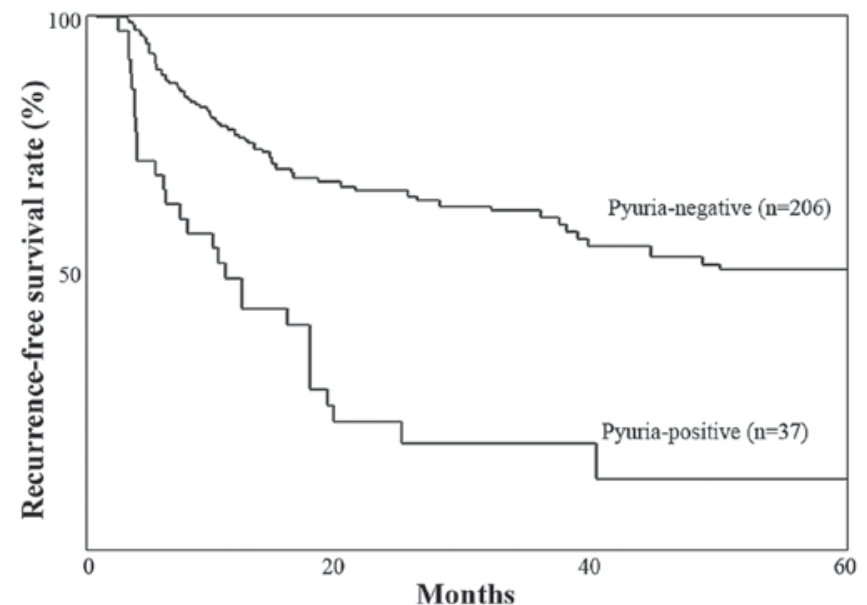

Figure 1. Kaplan-Meier recurrence-free survival (RFS) curves for bladder cancer patients receiving bacillus Calmette-Guérin therapy without pyuria $(n=206)$ vs. those with pyuria $(n=37)$. The 3 -year RFS rate was 62 vs. $20 \%$ in pyuria-negative vs. pyuria-positive cases, respectively; $\mathrm{P}<0.0001$ (log-rank test).

Table I. Characteristics of patients receiving intravesical BCG treatment $(n=243)$.

Characteristics

No. of patients (\%)

Age (years)
$<80$
$\geq 80$

Gender

Male

Female

Pyuria (no. of WBC/hpf)

$\leq 9$

$206(85)$

$\geq 10$

No. of tumors

1

$2-7$

$\geq 8$

Tumor size $(\mathrm{cm})$

$<3$

$\geq 3$

T stage

Ta

$\mathrm{T} 1$

Carcinoma in situ

No

Yes

Grade

G1

G2

G3

$45(19)$

BCG, bacillus Calmette-Guérin; WBC, white blood cell; hpf, high-power field. 
Table II. Univariate and multivariate analysis of recurrence following intravesical BCG treatment.

\begin{tabular}{|c|c|c|c|c|}
\hline \multirow[b]{2}{*}{ Variables } & \multicolumn{2}{|c|}{ Univariate analysis } & \multicolumn{2}{|c|}{ Multivariate analysis } \\
\hline & $\mathrm{HR}(95 \% \mathrm{CI})$ & P-value & $\mathrm{HR}(95 \% \mathrm{CI})$ & P-value \\
\hline Age (years) & & & & \\
\hline$<80$ vs. $\geq 80$ & $2.26(1.50-3.35)$ & 0.0002 & $1.89(1.22-2.87)$ & 0.005 \\
\hline $\begin{array}{l}\text { Gender } \\
\text { Male vs. female }\end{array}$ & $1.56(1.01-2.38)$ & 0.0493 & & 0.309 \\
\hline $\begin{array}{l}\text { Pyuria (cells/hpf) } \\
\leq 9 \text { vs. }>9\end{array}$ & $3.36(2.17-5.08)$ & $<0.0001$ & $3.05(2.06-4.84)$ & $<0.0001$ \\
\hline No. of tumors & & & & \\
\hline $\begin{array}{l}\text { Single vs. } 2-7 \\
2-7 \text { vs. }>7\end{array}$ & $\begin{array}{l}1.51(1.03-2.25) \\
2.23(1.08-4.15)\end{array}$ & 0.0038 & $\begin{array}{l}1.25(1.02-1.76) \\
2.80(1.32-5.38)\end{array}$ & 0.0153 \\
\hline $\begin{array}{l}\text { Tumor size } \\
\leq 3 \text { vs. }>3 \mathrm{~cm}\end{array}$ & $3.75(1.93-6.50)$ & 0.0004 & & 0.124 \\
\hline $\begin{array}{l}\text { T stage } \\
\text { Ta vs. T1 }\end{array}$ & $2.47(1.61-6.55)$ & 0.0351 & & 0.854 \\
\hline $\begin{array}{l}\text { Carcinoma in situ } \\
\text { No vs. yes }\end{array}$ & & 0.254 & & 0.800 \\
\hline $\begin{array}{l}\text { Grade } \\
\text { G1 vs. G2 } \\
\text { G2 vs. G3 }\end{array}$ & $\begin{array}{l}1.66(1.08-2.62) \\
1.29(1.03-1.54)\end{array}$ & 0.0492 & & 0.247 \\
\hline
\end{tabular}

BCG, bacillus Calmette-Guérin; hpf, high-power field; HR, hazard ratio; CI, confidence interval.

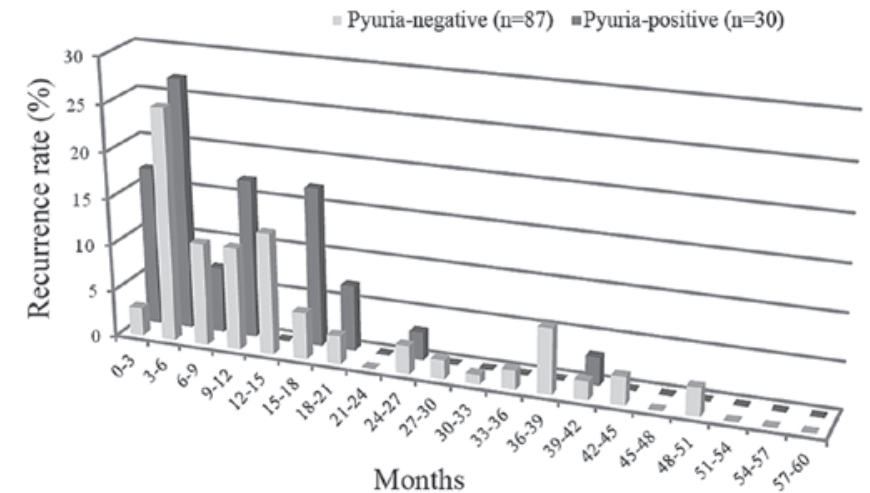

Figure 2. Distribution of time-to-recurrence. The patients resistant to bacillus Calmette-Guérin therapy were 87 among pyuria-negative and 30 among pyruia-positive patients. Pyuria-positive patients exhibited earlier recurrence.

Intravesical BCG treatment is effective for NMIBC, particularly for high- and intermediate-risk tumors (12). Although the precise mechanism of BCG immunotherapy is not known, previous studies have suggested that the response to BCG is dependent upon immunological reaction (13). A high degree of leukocyte infiltration is commonly observed in cancer-related inflammation. Recent studies have reported that inflammation within the tumor microenvironment may be associated with poor prognosis $(14,15)$. Therefore, we hypothesized that tumor-associated inflammation may exert a detrimental effect on BCG treatment and lead to eventual tumor recurrence. Pyuria is a established marker of inflammation. In this study, we assessed the presence of pyuria at the time of tumor recurrence and observed that pyuria independently predicted response to $\mathrm{BCG}$.

The present study demonstrated that NMIBC with pyuria exhibited a higher overall recurrence rate compared with NMIBC without pyuria. It was previously demonstraded that BCG treatment may decrease early recurrence and maintain a low recurrence rate up to 800 days (16). In the pyuria-positive group, early recurrence (within 6 months) was observed more frequently, whereas late recurrence (after 24 months) was observed in only 1 case (Fig. 2). These findings suggest that bladder cancers with pyuria are resistant to BCG therapy.

There are a number of possible explanations for this association between BCG resistance and pyuria. First, since bladder tumors may grow in an inflammatory environment, cancer cells are already resistant to immune cells. Additionally, the inflammation induced by the bladder tumor may inhibit the antitumor immune reaction produced by BCG treatment. In this immune escape, the neutrophils or other immune cells, including macrophages, regulatory $\mathrm{T}$ cells, or MDSCs, recruited through the inflammatory process, may be the key players (6). However, further investigation is warranted.

The limitations of the present study included the small number of patients, the retrospective nature of the analysis, conducted via medical chart review, and that the regimen of BCG was not the currently standard regimen. Recently, the efficacy of maintenance treatment with BCG has been reported (17). In the present study, all the cases only received 
8 cycles of weekly intravesical instillations and no maintenance administration of BCG.

We compared the efficacy of intravesical instillation therapy with BCG following TURBT between NMIBC patients with pyuria and those without pyuria. BCG treatment suppressed recurrence in pyuria-negative patients. Therefore, the presence of pyuria may be an independent predictor for BCG therapy according to the multivariate analysis. The prophylactic effect of BCG during the early phase (within 8 months) was weak in the pyuria-positive group.

\section{References}

1. Brake M, Loertzer H, Horsch R and Keller H: Recurrence and progression of stage $\mathrm{T} 1$, grade 3 transitional cell carcinoma of the bladder following intravesical immunotherapy with bacillus Calmette-Guérin. J Urol 163: 1697-1701, 2000.

2. Brake M, Loertzer H, Horsch R and Keller H: Long-term results of intravesical bacillus Calmette-Guérin therapy for stage T1 superficial bladder cancer. Urology 55: 673-678, 2000.

3. Lamm DL, van der Meijden PM, Morales A, Brosman SA Catalona WJ, Herr HW, Soloway MS, Steg A and Debruyne FM: Incidence and treatment of complications of bacillus Calmette-Guérin intravesical therapy in superficial bladder cancer. J Urol 147: 596-600, 1992.

4. Lima L, Dinis-Ribeiro M, Longatto-Filho A and Santos L: Predictive biomarkers of bacillus Calmette-Guérin immunotherapy response in bladder cancer: Where are we now? Adv Urol 2012: 232609, 2012.

5. Coussens LM and Werb Z: Inflammation and cancer. Nature 420: 860-867, 2002

6. Whiteside TL: The tumor microenvironment and its role in promoting tumor growth. Oncogene 27: 5904-5912, 2008.

7. Azuma T, Nagase Y and Oshi M: Pyuria predicts poor prognosis in patients with non-muscle-invasive bladder cancer. Clin Genitourin Cancer 11: 331-336, 2013.
8. Echols RM, Tosiello RL, Haverstock DC and Tice AD: Demographic, clinical, and treatment parameters influencing the outcome of acute cystitis. Clin Infect Dis 29: 113-119, 1999.

9. Mostofi FK, Sobin LH and Tosoni I: Histological Typing of Urinary Bladder Tumours. International Histological Classification of Tumours, No 19. World Health Organisation, Geneva, 1973.

10. Nieder AM, Brausi M, Lamm D, O'Donnell M, Tomita K, Woo H and Jewett MA: Management of stage T1 tumors of the bladder: International Consensus Panel. Urology 66 (Suppl 1): 108-125, 2005.

11. Hagemann T, Balkwill $\mathrm{F}$ and Lawrence T: Inflammation and cancer: A double-edged sword. Cancer Cell 12: 300-301, 2007.

12. Millán-Rodríguez F, Chéchile-Toniolo G, Salvador-Bayarri J, Palou J, Algaba F and Vicente-Rodríguez J: Primary superficial bladder cancer risk groups according to progression, mortality and recurrence. J Urol 164: 680-684, 2000.

13. Zuiverloon TC, Nieuweboer AJ, Vékony H, Kirkels WJ, Bangma $\mathrm{CH}$ and Zwarthoff EC: Markers predicting response to bacillus Calmette-Guérin immunotherapy in high-risk bladder cancer patients: A systematic review. Eur Urol 61: 128-145, 2012.

14. Donskov F and von der Maase H: Impact of immune parameters on long-term survival in metastatic renal cell carcinoma. J Clin Oncol 24: 1997-2005, 2006

15. Ilie M, Hofman V, Ortholan C, Bonnetaud C, Coëlle C, Mouroux J and Hofman P: Predictive clinical outcome of the intratumoral CD66b-positive neutrophil-to-CD8-positive T-cell ratio in patients with resectable nonsmall cell lung cancer. Cancer 118: 1726-1737, 2012

16. Hinotsu S, Akaza H, Isaka S, et al; BCG Tokyo 172 Strain Study Group: Sustained prophylactic effect of intravesical bacille Calmette-Guérin for superficial bladder cancer: A smoothed hazard analysis in a randomized prospective study. Urology 67: 545-549, 2006.

17. Lamm DL, Blumenstein BA, Crissman JD, et al: Maintenance bacillus Calmette-Guérin immunotherapy for recurrent TA, T1 and carcinoma in situ transitional cell carcinoma of the bladder: A randomized Southwest Oncology Group Study. J Urol 163: 1124-1129, 2000. 